\title{
SHEAR BOND STRENGTH OF AGED MONOLITHIC ZIRCONIA VENEERS USING DIFFERENT TYPES OF BONDING AGENT
}

\author{
Ghada Abd El Fattah", Tarek Salah El Din Morsy"* and Dina Salah Ibrahim Afifi**
}

\begin{abstract}
Objective: The purpose of this study was to evaluate the effect of MDP containing resin cement and surface treatment of Translucent zirconia and Ultra translucent zirconia on shear bond strength.

Material \& Methods: Eighty zirconia discs were constructed . Samples were divided into two groups: Group I: Translucent zirconia samples (n=20) Gro up II: Ultra translucent zirconia samples ( $\mathrm{n}=20$ ) Each group will be subdivided into 2 subgroups according to the surface treatment Subgroup A: Samples will be treated with air abrasion Sub group B: Samples will be left untreated. Each sub group will be further subdivided into 2 subgroups according to bond type: Subgroup 1: samples cemented with MDP containing bond (Universal bond). Subgroup 2: samples cemented with non MDP containing bond (Adper single bon II). All tested samples were subjected to Shear bond strength test.Deboneded samples were examined under the scan electron microscope to determine their mode of failure.Data were statistically analyzed and recorded.
\end{abstract}

Results: Regarding the effect of ceramic material and resin cement :For MDP containing resin cement, translucent zirconia had a significantly higher value than ultra translucent zirconia. While for Non-MDP containing resin cement there was no significant difference between both materials. Regarding the effect of surface treatment and resin cement for both treated and untreated samples, MDP containing resin cement had a significantly higher value than Non-MDP containing resin cement.

Conclusion: the use of phosphate monomer luting cements on freshly air-abraded zirconia as the simplest and most effective way for zirconia cementation procedure.

KEYWORDS: Zirconia Veneers; MDP.

\section{INTRODUCTION}

Metal free ceramic restoration have become the most commonly used restorations in fixed prosthodontics nowadays. All ceramic restorations offers a wide variety of materials with wide range of uses, among these category of restoration zirconiumdioxide prevaled as the strongest among these groups. Zirconium dioxide ceramics offer

\footnotetext{
* Lecturer, Fixed Prosthodontics Department, Faculty of Dentistry, Ain Shams University

** Professor, Fixed Prosthodontics Department, Faculty of Dentistry, Ain Shams University
} 
a wide variety of clinical applications. ${ }^{1}$ The flexural strength of zirconia is 900 to $1200 \mathrm{MPa}$, fracture resistance of more than $2000 \mathrm{~N}$, and fracture toughness of 9-10 $\mathrm{MPam}^{0.5}$, which is almost twice that of alumina based materials. CAD/CAM technologies have made working with this high crystalline material simpler, allowing the fabrication of full coverage crowns, bridge framework ,implant based restoration and full arches restoration with the least effort. ${ }^{2}$

The main disadvantages of such material is their lack of translucency and their tenacious need for veneering. Recently, translucent and ultra translucent zirconia has been introduced these materials combines between the high strength and the good esthetics. To increase the translucency of any material, the translucency parameter must be increased and the the contrast ratio must be decreased. ${ }^{3}$

The ultra translucent material is more translucent than the standard zirconia because it has more cubic form more than the tetragonal form,this is done by adding $5 \mathrm{~mol} \%$ yttria. The standard zirconia formula $53 \%$ cubic form and $47 \%$ tetragonal form as this combination leads to increase the translucency for about $19 \% .^{4}$

Conventional cementation techniques with zirconium dioxide components do not provide sufficient bond strength. Strong resin bonding depends on micromechanical interlocking and adhesive chemical bonding to the ceramic surface, which requires surface roughening for mechanical bonding and surface activation for chemical adhesion. $^{5}$

Surface of zirconia is not able to be etched by a low concentration of HF. Unlike in the use of other existing ceramics, the use of silane was reported to be ineffective due to the absence of silica components. Mechanical or chemical methods have been attempted for a stable bonding between zirconia and resin cement ${ }^{6}$. In order to increase the mechanical bonding force by making the fine irregular structure, airborne particle abrasion used. A primer or cement containing 10-methacryloyloxydecyl dihydrogen phosphate (10-MDP) monomer has been used for chemical bonding. The phosphate ester group of the MDP was reported to directly bond to metal oxide ${ }^{7}$. Another reaction might have been formed between the hydroxyl group in the MDP monomer and the hydroxyl group on the zirconia surface ${ }^{8}$. But it is not obvious whether there is a true chemical bond with zirconia or there is a micro-retentive bond promoted by sand blasting. ${ }^{9}$

In order to simplify adhesive cementation procedures, self-adhesive resin cements were developed, they require fewer clinical steps..$^{10}$ Various self adhesive resin cements consist of phosphate monomers, including MDP, and manufactures suggest that clinicians apply self adhesive cements to Y-TZP without additional Y-TZP primer ${ }^{11}$. Thus, it is necessary to evaluate the bond strength between the MDP containing self adhesive resin cements and Y-TZP ceramics. ${ }^{12}$

So it was highly advocated to study this bond in more detailes in order to reach cognitive understanding of its nature and the factor affecting it.

\section{MATERIALS AND METHODS}

\section{A) Materials}

The main materials were used in this studty were translucent zirconia (Bruxzir), and ultra translucent zirconia (Bruxzir Anterior). Rely x veneer light cure cement, Scotch bond universal adhesive: Adper single bond 2:

\section{B) Methodolgy}

Eighty zirconia ceramic samples were constructed with standard dimensions for this study.

Zirconia samples were examined by SEM and adhesive resin cement bonded to zirconia discs test the effect of different surface treatments of the zirconia ceramic discs on the shear bond strength. 


\section{Technique of cutting of zirconia samples}

The Roland Milling Unit was used to millzirconia blank to produce blocks of translucent zirconia and ultra translucent zirconia, with the following dimensions (12 mm length, $12 \mathrm{~mm}$ diameter). Blocks were cut using ISOMET4000 with diamond blade with speed of (2150 rpm) (thickness $0.6 \mathrm{~mm}$, diameter $12 \mathrm{~mm})$. Zirconia discs were cut in larger dimensions to compensate for the shrinkage during the sintering stage all samples were checked for uniform thickness by digital cailper after sintering showing thickness $0.5 \mathrm{~mm}$ and 10 in diameter. All zirconia samples were placed in a sintering furnace(Nabertherm), and sintered for 9 hours at temperature $1580^{\circ} \mathrm{C}$ for translucent zirconia samples, and $1530^{\circ} \mathrm{C}$ ultra translucent zirconia samples according to manufacturer instructions. Zirconia samples were finished by using silicon carbide grinding paper 600 grit. Samples were ultrasonically cleaned in distelled water in ultra sonic cleaning device for 190 seconds, and then left to bench dry.

\section{Formation of zirconia base}

Epoxy resin base were made for holding zirconia discs. Molds were made from pvc water tubes were cut into $25 \mathrm{~mm}$ internal diameter and $20 \mathrm{~mm}$ length with especially fabricated teflon cover. zirconia discs are placed over teflon cover then epoxy resin is poured over the zirconia discs then left to set, teflon cover is removed, epoxy resin base is finished for excess removal.

\section{Surface treatment of zirconia}

Air abrasion of zirconia: 20 samples of translucent zirconia and 20 samples of ultratranslucent zirconia were treated with sandblasting. Sandblasting was done using a sandblasting machine with $\mathrm{AL}_{2} \mathrm{O}_{3}$ particles of $50 \mu \mathrm{m}$ size at an angle $90^{\circ}$, distance $10 \mathrm{~mm}$ for 15 seconds and 2.5 bar pressure and samples were moved in horizontal direction to make sure that all surfaces were treated. Cleaning of samples have been done for 30 minutes and left to bench dry.

Samples of each group were examined by scanning electron microscope before and after surface treatment. Samples were magnified up to 5000 magnifications. SEM was used to evaluate the topographic patterns before and after surface treatment.

\section{Application of resin cement}

40 discs of zirconia samples were treated by application of universal single bond by bond brush for 20 seconds then air dried by gentle oil free air for 5 seconds without curing. The other 40 discs of zirconia samples were treated by appliction of adper single bond 2 by bond brush for 20 seconds then air dried by gentle oilfree air for 5 seconds without curing. A catheter tube was prepared with lancet into $5 \mathrm{~mm}$ length $9 \mathrm{~mm}$ diameter and bonded onto the free surface of zirconia samples then cured for 30 seconds. Rely x veneer cement has been applied into the tube.The excess cement was then removed by dry cotton pellet then the cement was light cured for 30 secondsby LED curing* unit circumferentially at $650 \mathrm{~W} / \mathrm{cm}^{2}$ intensity according to manufacturer's instructions_

\section{Thermo cycling aging}

Thermocycling was done under the following conditions: 5000 cycle at the $5{ }^{\circ} \mathrm{C}$ and $55^{\circ} \mathrm{C}$ thermal circulation water baths. ${ }^{13}$

\section{Shear bond strength test technique}

Test procedure: All samples were individually and vertically mounted on a computer controlled material testing machine with loaded $5 \mathrm{kN}$ and data were recorded using computer software. Shear test was done by applyingcompressive mode of load at zirconia composite interface until failure occurs,

\footnotetext{
* Wood Pecker, China.
} 
using a mono beveled chisel shaped metallic rod with size $6 \mathrm{~mm}$ and angle $90^{\circ}$.

Shear bond strength calculation: The load of failure was divided by bonding area to express the bond strength in MPa.Debonded samples were examined by the scan electron microscope to determine their mode of failure.

\section{Statistical analysis:}

Numerical data were explored for normality by checking the data distribution, calculating the mean and median values and using Kolmogorov-Smirnov and Shapiro-Wilk tests. Data showed parametric distribution so; it was represented by mean and standard deviation (SD) values. Three-way ANOVA was used to study the effect of different tested variables and their interactionon shear bond strength. Independent t-test was used to analyzesimple main effects.The significance level was set at $\mathrm{P} \leq 0.05$ for all tests. Statistical analysis was performed with IBM ${ }^{\circledR}$ SPSS $®$ Statistics Version 25 for Windows.

\section{RESULTS}

\section{Effect of different variables and their interaction}

Effect of different variables and their interaction on Shear bond strength (Mpa) were presented in table (1)

There was a significant interaction between type of resin cement and both the ceramic material and the surface treatment.

\section{Effect of ceramic material and resin cement}

For both materials, MDP containing adhesive had a significantly higher $($ mean \pm SD) value than Non-MDP containing adhesive.

\section{Effect of surface treatment and bonding agent}

For treated and untreated samples, MDP containing adhesive had a significantly higher $($ mean \pm SD) value than Non-MDP containing adhesive.

TABLE (1) Effect of different variables and their interactions on Shear bond strength (Mpa)

\begin{tabular}{|l|c|c|c|c|c|}
\hline \multicolumn{1}{|c|}{ Source } & Type III Sum of Squares & df & Mean Square & F & P-value \\
\hline Corrected Model & $186.621^{\mathrm{a}}$ & 7 & 26.660 & 75.580 & $<0.001^{*}$ \\
\hline Intercept & 392.923 & 1 & 392.923 & 1113.919 & $<0.001^{*}$ \\
\hline Ceramic material & 3.626 & 1 & 3.626 & 10.280 & $0.002^{*}$ \\
\hline Surface treatment & 9.881 & 1 & 9.881 & 28.013 & $<0.001^{*}$ \\
\hline Adhesive & 166.563 & 1 & 166.563 & 472.199 & $<0.001^{*}$ \\
\hline Material* Treatment & 1.086 & 1 & 1.086 & 3.079 & $0.084 \mathrm{~ns}$ \\
\hline Material* adhesive & 2.258 & 1 & 2.258 & 6.401 & $0.014^{*}$ \\
\hline Treatment*adhesive & 2.053 & 1 & 2.053 & 5.819 & $0.018^{*}$ \\
\hline Material* Treatment*adhesive & 1.154 & 1 & 1.154 & 3.272 & $0.075 \mathrm{~ns}$ \\
\hline Error & 25.397 & 72 & 0.353 & & \\
\hline Total & 604.941 & 80 & & & \\
\hline Corrected Total & 1859.449 & 19 & & & \\
\hline
\end{tabular}


TABLE (2) Mean \pm standard deviation (SD) of Shear bond strength (Mpa) for different ceramic materials and adhesive

\begin{tabular}{|c|c|c|c|}
\hline \multirow[b]{2}{*}{ Ceramic material } & \multicolumn{2}{|c|}{ Adhesive (mean \pm SD) } & \multirow[b]{2}{*}{ P-value } \\
\hline & $\begin{array}{c}\text { MDP } \\
\text { containing }\end{array}$ & $\begin{array}{l}\text { Non-MDP } \\
\text { containing }\end{array}$ & \\
\hline Translucent zirconia & $4.04 \pm 1.13$ & $0.81 \pm 0.33$ & $<0.001 *$ \\
\hline $\begin{array}{l}\text { Ultra translucent } \\
\text { zirconia }\end{array}$ & $3.27 \pm 0.77$ & $0.72 \pm 0.29$ & $<0.001 *$ \\
\hline P-value & $0.018^{*}$ & $0.373 \mathrm{~ns}$ & \\
\hline
\end{tabular}

Significant $(p \leq 0.05) n s ;$ non-significant $(p>0.05)$

TABLE (3) Mean \pm standard deviation (SD) of Shear bond strength (Mpa) for different surface treatments and adhesive

\begin{tabular}{|c|c|c|c|}
\hline \multirow{2}{*}{$\begin{array}{c}\text { Surface } \\
\text { treatment }\end{array}$} & \multicolumn{2}{|c|}{ Adhesive (mean \pm SD) } & \multirow[b]{2}{*}{ P-value } \\
\hline & $\begin{array}{c}\text { MDP } \\
\text { containing }\end{array}$ & $\begin{array}{l}\text { Non-MDP } \\
\text { containing }\end{array}$ & \\
\hline Air abrasion & $4.17 \pm 1.12$ & $0.96 \pm 0.29$ & $<0.001 *$ \\
\hline Untreated & $3.14 \pm 0.60$ & $0.58 \pm 0.19$ & $<0.001 *$ \\
\hline P-value & $0.001 *$ & $<0.001 *$ & \\
\hline
\end{tabular}

*; significant $(p \leq 0.05)$ ns; non-significant $(p>0.05$

\section{Effect of ceramic material and surface treatment}

For both materials, Air abraded samples had a non-significantly higher $($ mean $\pm \mathrm{SD})$ value than untreated samples.

TABLE (4) Mean \pm standard deviation (SD) of Shear bond strength (Mpa) for different ceramic material and surface treatments.

\begin{tabular}{|l|c|c|c|}
\hline \multirow{2}{*}{ Ceramic material } & \multicolumn{2}{|c|}{$\begin{array}{c}\text { Surface treatment } \\
\text { (mean } \pm \text { SD) }\end{array}$} & \multirow{2}{*}{ P-value } \\
\cline { 2 - 3 } & Air abrasion & Untreated & \\
\hline Translucent zirconia & $2.89 \pm 2.08$ & $1.96 \pm 1.42$ & $0.106 \mathrm{~ns}$ \\
\hline $\begin{array}{l}\text { Ultra translucent } \\
\text { zirconia }\end{array}$ & $2.23 \pm 1.47$ & $1.76 \pm 1.34$ & $0.299 \mathrm{~ns}$ \\
\hline P-value & $0.256 \mathrm{~ns}$ & $0.663 \mathrm{~ns}$ & \\
\hline
\end{tabular}

*; significant $(p \leq 0.05) n s ;$ non-significant $(p>0.05)$

\section{Scanning Electron Microscopic Results:}

Scan electron microscopic examination has been done of surface topography following different surface treatments in comparison with the control group. (figure 1, 2)

\section{Before surface treatment}

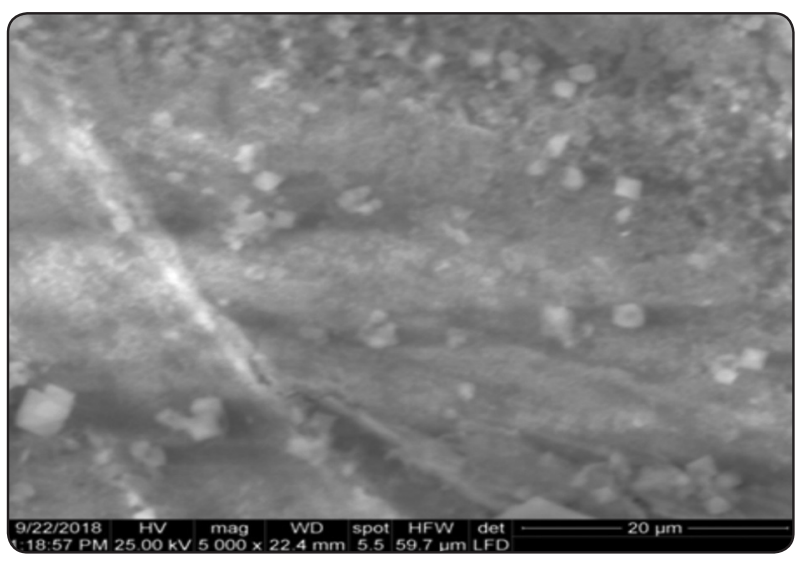

Fig. (1) Translucent zirconia

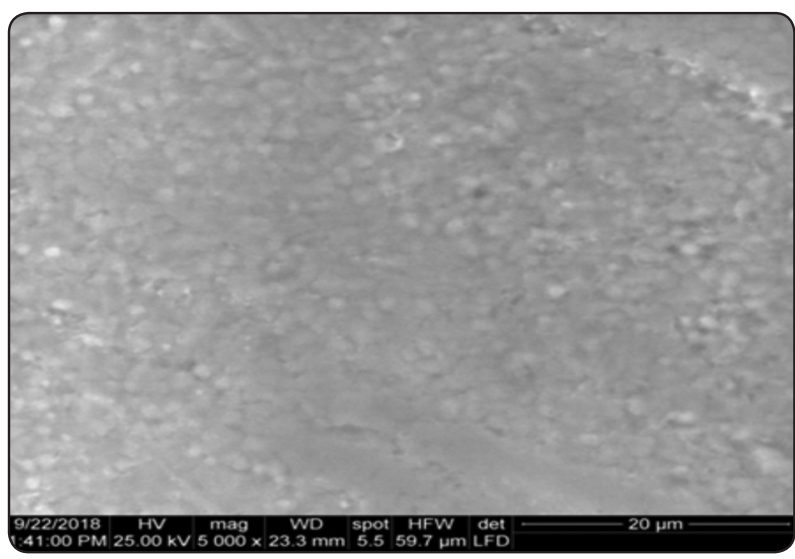

Fig. (2) Ultra translucent zirconia

\section{After Air Abrasion:}

On viewing SEM photographs of samples that has no treatment at all and samples that has been treated by $\mathrm{A} 12 \mathrm{O} 3$ Air abrasion at 5000X Magnifications, a slightly irregular surface was revealed together with the evidence of elevation and depressions that allow flow of adhesive between them and the micromechanical retention which 
has been embedded on the surface for Translucent zirconia samples (figure3, 4) and Ultra translucent zirconia samples compared to non-treated samples.

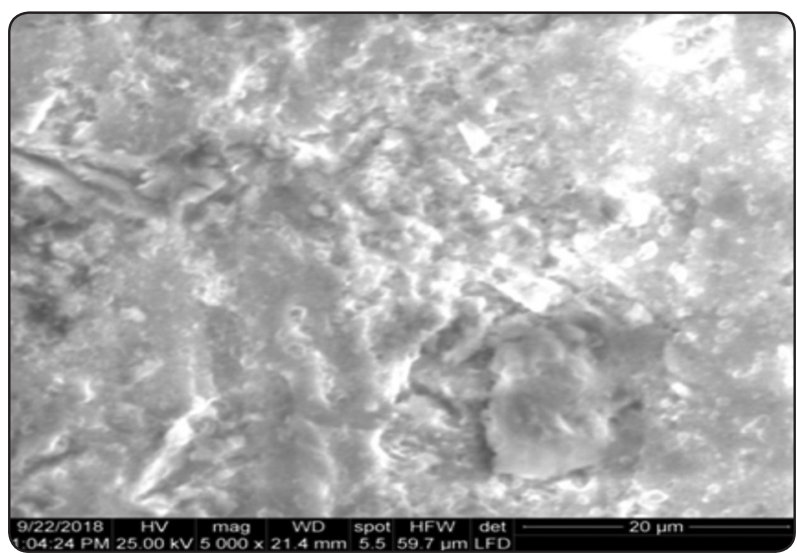

Fig. (3) Translucent zirconia

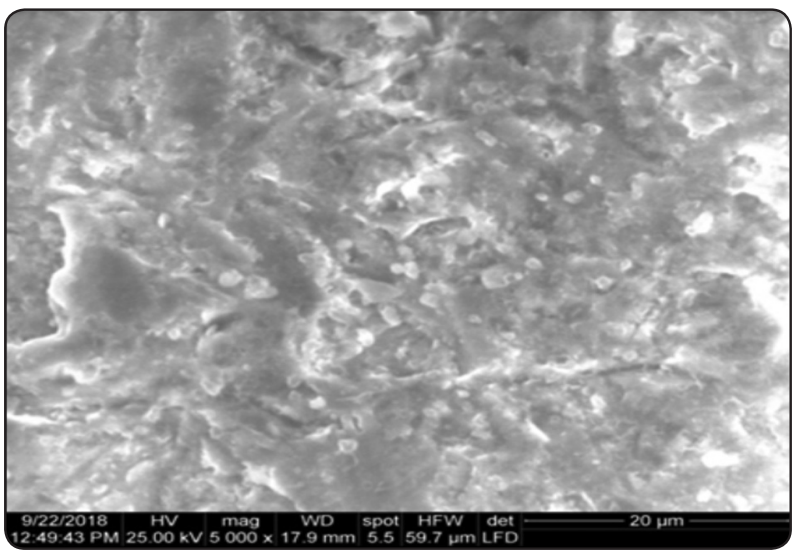

Fig. (4) Ultra translucent zirconia

\section{After debonding:}

Samples of each group were examined after debonding by SEM that has been used to evaluate cement line and mode of failure. All of samples were magnified up to $800 \mathrm{X}$.

Regarding debonded Translucent and Ultra translucent zirconia samples with air abrasion and MDP containing resin cement showed mixed type of failures with tendency toward the adhesive failure behavior since cement line was covering $<30 \%$ of zirconia suface(figure $5,6,7$ ).

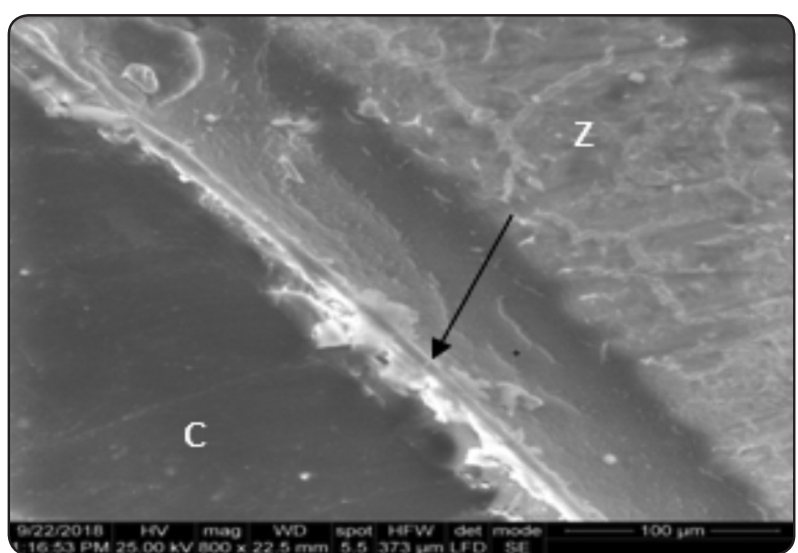

Fig. (5) SEM Translucent zirconia with air abrasion and MDP afterdebonding. Debonded samples of air abrasion translucent zirconia sample with cement layer on < $30 \%$ of the surface, represented with dark grey area

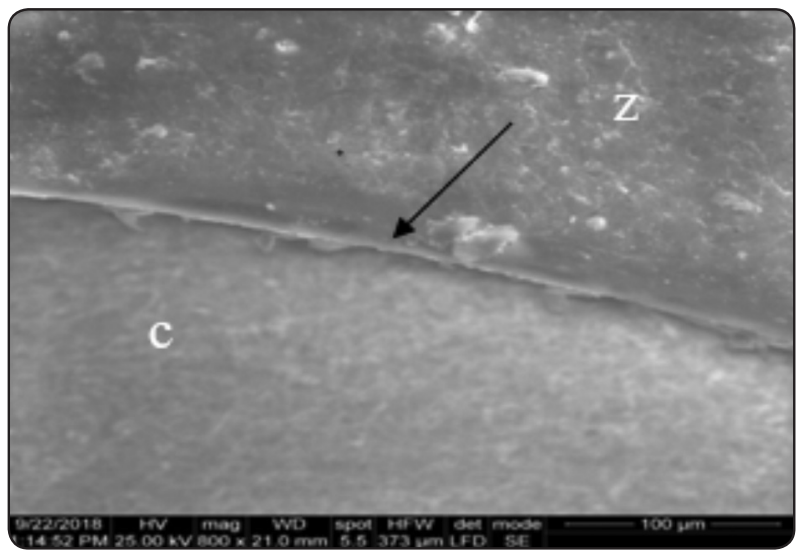

Fig. (6) SEM Translucent zirconia(z) with air abrasion and no MDP after debonding-Debonded translucent zirconia sample with cement layer on on $<30 \%$ of the surface showed by arrow, represented with dark gray color.(c)

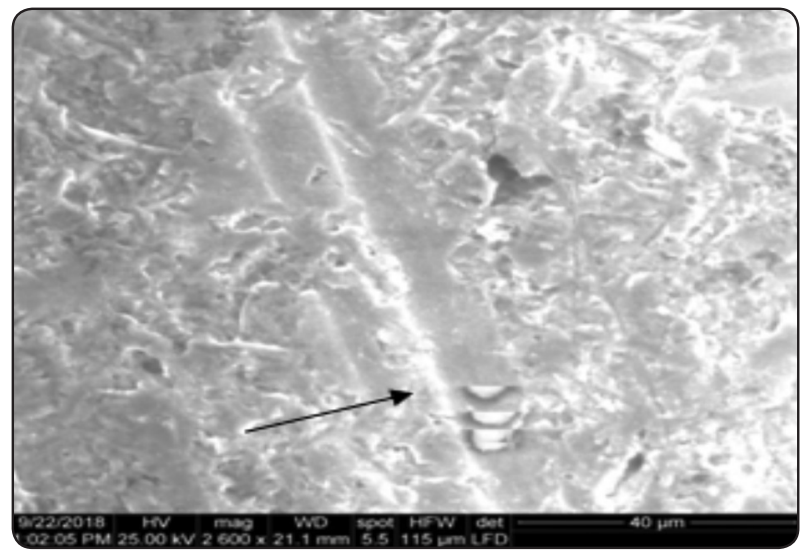

Fig. (7) Ultra translucent zirconia with MDP and air abrasion after debonding. Debonded samples with evidence of cement layer showed by arrow on $<30 \%$ of the surface 
Regarding debonded Translucent and Ultra translucent zirconia samples with no air abrasion and no MDP containing resin cement showed mixed type of failures with tendency toward the cohesive failure behavior since cement line was covering $>60 \%$ of zirconia sufaceand the presence of microcracks and micro pores (figure8,9,10,11,12).

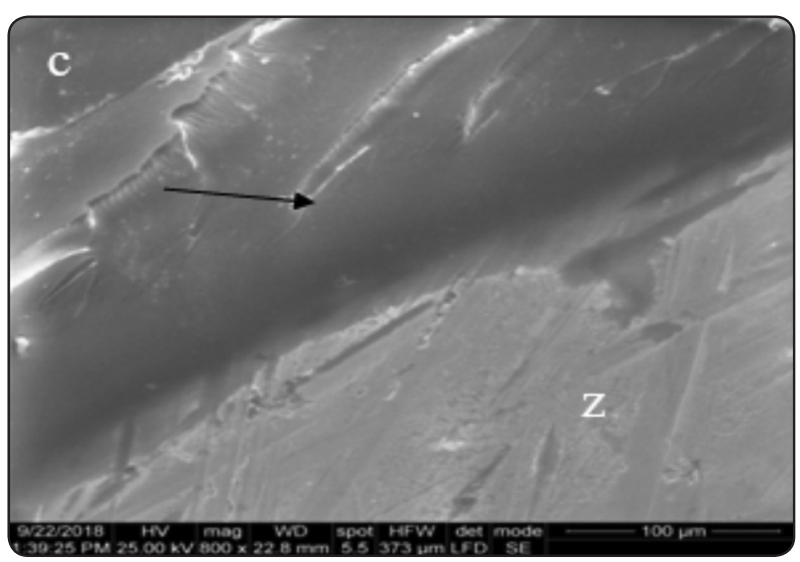

Fig. (8) SEM Translucent zirconia with MDP and no air abrasion after debonding. Cement layer of deboned zirconia samples showing evidence of microcracks that shows adhesive failure

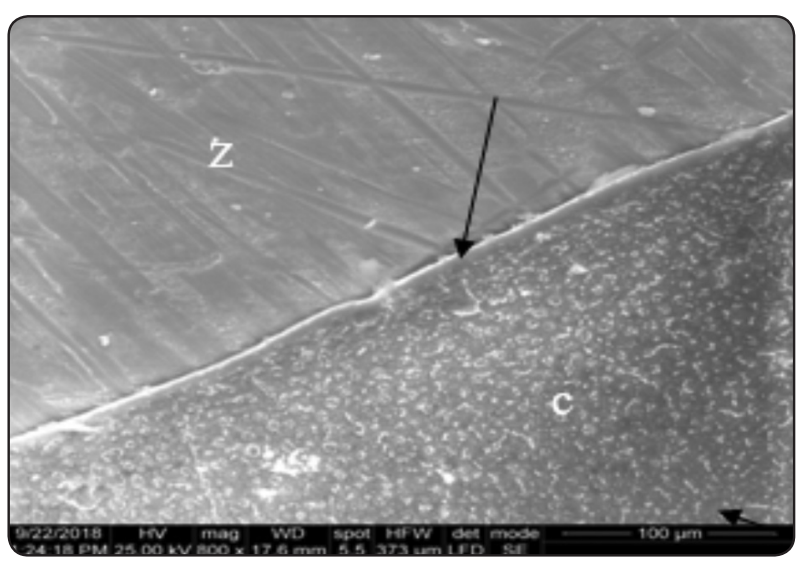

Fig. (9) SEM Translucent zirconia(z) with no MDP and no air abrasion after debonding adhesive failure in cement layer showed by arrow with presence of multiple micropores( c)

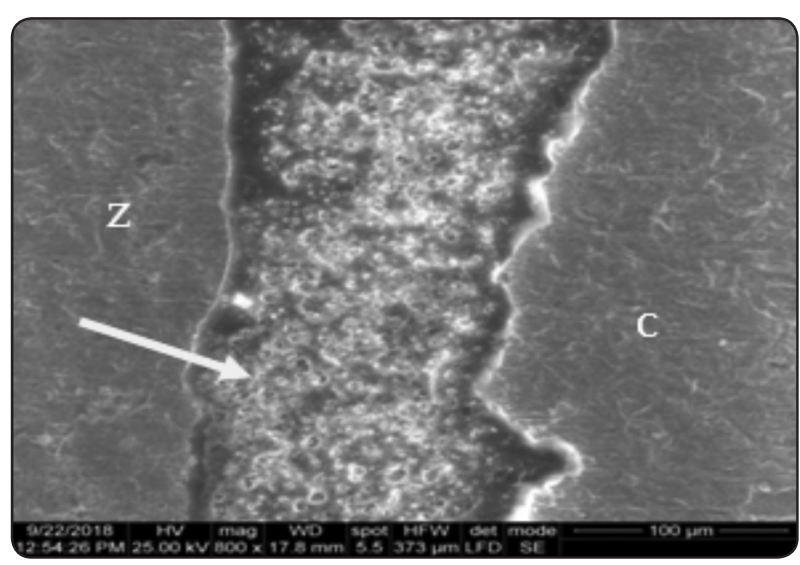

Fig. (10) Ultra translucent zirconia(z) with no MDP and air abrasion after debonding cement layer represents $>70 \%$ with evidence of multiple micropores showed by arrow

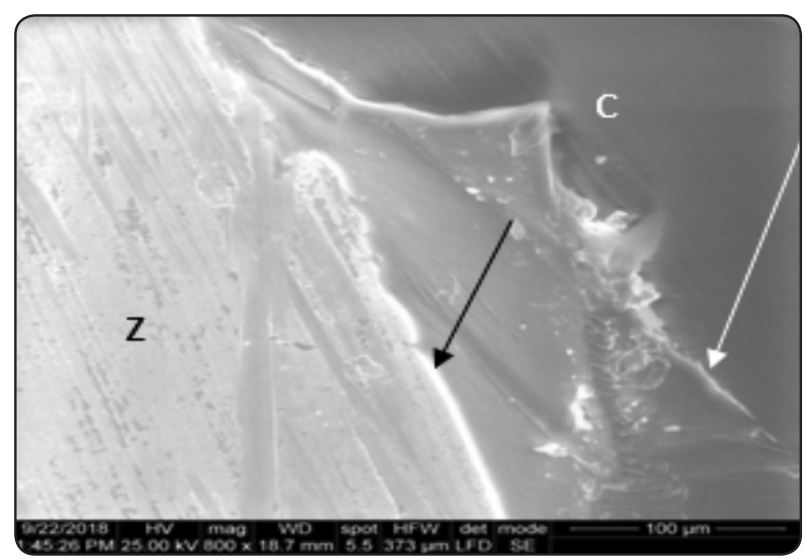

Fig. (11) Ultra translucent zirconia ( $\mathrm{z}$ ) with MDP and no air abrasion after debonding cement line represents $>70 \%$ represented by black arrow with evidence of multiple micrcracks represented by white arrow and micro pores in the cement area with dark grey color(c).

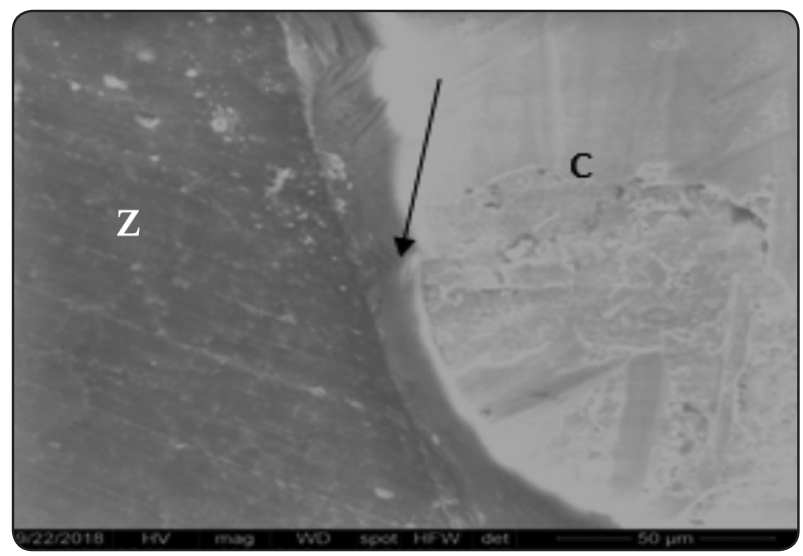

Fig. (12) Ultratranslucent zirconia (z)with no MDP and no air abrasion after debonding. Debonded translucent zirconia sample with cement layer on $>60 \%$ of the surface represented by arrow, represented with dark gray color(c). 


\section{DISCUSSION}

Zirconia ceramic has attracted attention in recent dental researches and clinical use, zirconia considered standard strength but achieving high translucency and strong bonding is always a matter of conflict.

The aim of the present study was to evaluate the effect of air abrasion $\left(\mathrm{Al}_{2} \mathrm{O}_{3}\right)$ sandblasting and the use of MDP monomer on the shear bond strength at the monolithic zirconia/ resin cement interfaces evaluated.

The samples in this study were cut using ISOMET 4000 precision saws cut materials to size of $16 \times 10$ x $0.5 \mathrm{~mm}$ uniformly following ISO -682, 2008 guidelines for sample preparation ${ }^{14}$ The thickness of zirconia blank considered the height of specimens, width and thickness of specimens were adjusted in the software of the cutting device. The thickness were chosen to resemble the average veneer thickness.

There is a general consensus that airborne particle abrasion with $50-110 \mu \mathrm{m}$ alumina particles at $0.25 \mathrm{MPa}$ is effective in roughening and cleaning the bonding surface of zirconia ${ }^{15}$ Air abrasion of zirconia has been done using $\mathrm{AL}_{2} \mathrm{O}_{3}$ particles of $50 \mu \mathrm{m}$ size.

Adhesive resin cement (Rely x veneer) has been applied to 80 discs of zirconia samples that have been treated by application of (adper single bond 2) which considers a non MDP containing bond as a control group and 40 zirconia discs have been treated with universal single bond which contains MDP (10-methacryloyl oxydecyl dihydrogen phosphate) that has phosphate monomer group. Fons-font et al. ${ }^{5}$ Reported that chemical bond between zirconia and resin cements can be improved by MDP (10-methacryloyloxydecyl dihydrogen phosphate

Thermocycling has been done under the following conditions: 5000 cycle at the $5{ }^{\circ} \mathrm{C}$ and $55^{\circ} \mathrm{C}$ thermal circulation water baths had been performed Yap et al. ${ }^{13}$ suggested that the 5000 cycles were clinically approximating six months of use inside patient mouth. All samples were subjected to thermo cycling for 5000 cycle.
All samples were subjected to shear testindividualy with $5 \mathrm{KN}$ load. Shear test was done by applying compressive mode of load at zirconia composite interface until failure occurs, using mono beveled chisel shaped metallic rod. Su et al. ${ }^{16}$ found that different methods have been used to evaluate bond strength of zirconia such as flexure strength tests, tensile strength test,and shear bond strength test. Shear test reported best results between zirconia and resin cement which yeilds more standerdized results.

\section{Concerening type of ceramic material}

Translucent zirconia recorded higher statistically non-significant difference mean bond strength value than ultra-translucent zirconia, probbaly Translucent zirconia has more tetragonal structure which contains more oxygen ions than cubic structure which is found more in ultra translucent zirconia also cubic structure considered yttria-rich region and a tetragonal structure considered yttria poor region this allows more affinity to MDP being more reactive with tetragonal strucure rather than the cubic one. ${ }^{17}$

Noriyuki Nagaoka evaluated the effects of MDP-based primers on shear bond strength between resin cement and zirconia on four brands of translucent zirconia they not only revealed ionic bonding but also hydrogen bonding between 10-MDP and Zirconia. ${ }^{18}$ this results support the current study.

\section{Concerning Air borne particle abrasion}

For both materials, air abraded samples had a non-significantly higher $($ mean \pm SD) value than untreated samples.. That was approved by others investigators who said that the absence of use particle abrasion as surface treatment would result in a dramatic reduction in bond strength. According to them, surface roughness is a key factor for adhesion to zirconia. ${ }^{19}$ Surface abrasion or roughening through airborne particle abrasion, establishes adhesion through micro-mechanical retention ${ }^{(20)} \mathrm{Nev}$ ertheless, airborne abrasion also cleans the surface, 
removes impurities, modifies the surface energy and wettability. ${ }^{21}$

The highest bond strength for translucent zirconia and ultra translucent zirconia with $\mathrm{Al}_{2} \mathrm{O}_{3}$ air abrasion method was explained by Ahn et al. (2015) which is thought to assist in the progress of adhesive flow into micro retentions due to increased roughness and surface energy, which create micromechanical interlocking between the adhesive and zirconia. Moreover, air abrasion may generate hydroxyl groups on the zirconia surfaces, facilitating the chemical reaction with phosphate monomers. ${ }^{12}$

Şafak Kulunk ${ }^{22}$ : evaluated the effect of air abrasion with different particles of different sizes and forms on the shear bond strength of adhesive resin cement to zirconia. The highest bond strengths were obtained by air abrasion with $30-50 \mu \mathrm{m}$ synthetic diamond particles.

\section{Concerning MDP bond}

For treated and untreated samples, MDP containing adhesive had a significantly higher (mean \pm SD) value than Non-MDP containing adhesive due to presence of Phosphate Monomer Regarding this study, Liang Chen and Byoung InSuh has evaluated silica based lithium disilicate and non- silicatebased Zirconia, It is still difficult to achieve a strong and durable resin ceramic adhesion, especially resin Zirconia bonding. Focusing on the latest resin bonding techniques (including surface treatment, priming and cementation) for dental all-ceramic materials, especially Zirconia and lithium disilicate ceramics. They demonstrated that a variety of surface roughness, priming and cementation methods are available to improve resin ceramic bonding. The preferred protocol for Zirconia resin bonding is the combination of surface roughness such as air abrasion and treatment with a phosphate containing Zirconia primer followed by cementation with non phosphate-containing resin cement. ${ }^{14}$

Jin-Soo Ahn: ${ }^{12}$ evaluated the effect of Phosphate Monomer Containing Primers this study revealed that for the untreated zirconia samples application of MDP resin cements shows higher value of bond strength more than none MDP containing adhesive. Which is compatible with this study.

Cavalcanti et al. ${ }^{23}$ : evaluated the influence of surface treatments and metal primers on the bond strength of resin cements to a yttrium-stabilized tetragonal zirconia (Y-TZP) ceramic. The three metal primers yielded a significant increase in bond strength, regardless of the surface treatment and resin cement. Adhesive failures were the most prevalent. Air abrasion with $\mathrm{A} 12 \mathrm{O} 3$ particles and the application of metal primers increased bond strength to Y-TZP surfaces for both resin cements. which is compatible with this study.

Park YYJAY et al. ${ }^{24}$ Advocated The effect of Sandblasting and Different Primers on Shear Bond Strength Between Yttria-tetragonal Zirconia Polycrystal Ceramic and a Self-adhesive Resin Cement. Z-PRIME Plus primer application after air-abrasion presented the best results for improving the bond strength between Y- TZP ceramic and self-adhesive resin cement.whish is compatible with this study.

The main limitation of this study is its in-vitro design, which doesn't allow the variable interacting factors found in the oral cavity. Also the use of one combination of surface treatnment and MDP ,other combinations may give different results.

Bonding to zirconia appears to be multifactorial process, cannot depend on mechanical or chemical but always need combination of both. So further studies with other surface combinations are needed in order to conclude an optimum bonding protocol for such material.

\section{CONCLUSION}

Within the limitation of this study the following conclusion can be withdrawn:

- Both surface topography and shear bond strength value of ceramic systems are affected by the type of material and surface treatment performed.

- Sandblasting with $50-\mu \mathrm{m}$ alumina particles combined with MDP showed a reliable bond with monolithic zirconia . 
- The use of phosphate monomer luting cements on freshly air-abraded zirconia can be considered the simplest and most effective way for monolithic zirconia cementation procedure.

\section{REFERENCES}

1. Lin J, Shinya A, Gomi H, Shinya A. Effect of Self-adhesive Resin Cement and Tribochemical Treatment on Bond Strength to Zirconia. Int J Oral Sci. 2010; 2(1):28-34.

2. Daou, EE. The Zirconia Ceramic: Strengths and Weaknesses. Open Dent J. 2014; 8:33-42.

3. Al-Juaila E, Osman E, Segaan L, Shrebaty M, Farghaly EA. Comparison of translucency for different thicknesses of recent types of esthetic zirconia ceramics versus conventional ceramics (in vitro study). Future Dent J. 2018;4(2):297-301.

4. Zhang Y. Making yttria-stabilized tetragonal zirconia translucent Dent. Mater. 2014 Oct ; 30(10) 1195-1203.

5. Fons-font A, Amigó-borrás V, Granell-ruiz M. Bond strength of selected composite resin-cements to zirconiumoxide ceramic.Med Oral Patol Oral Cir Bucal. 2013; 18(1):115-123.

6. Sriamporn T, Thamrongananskul N, Busabok C, Poolthong S, Uo M. Dental zirconia can be etched by hydrofluoric acid. Dental Materials Journal 2014;33(1):79-85.

7. Uo M, Gren GSJ, Sundh A, Goto M, Watari F, Bergman M. Effect of Surface Condition of Dental Zirconia Ceramic (Denzir) on Bonding. Dent Mater J. 2006:626-631.

8. Lee J, Lee C. Effect of the Surface Treatment Method Using Airborne-Particle Abrasion and Hydrofluoric Acid on the Shear Bond Strength of Resin Cement to Zirconia. Dent J (Basel). 2017.

9. Gargari M, Gloria F, Napoli E, Pujia AM. Zirconia: cementation of prosthetic restoration.Literature review. Oral Implantol (Rome). 2010: 3(4)25-29.

10. Aguiar TR, Francescantonio M Di, Ambrosano GMB, Giannini M. Effect of Curing Mode on Bond Strength of Self-Adhesive Resin Luting Cements to Dentin. J Biomed Mater Res B Appl Biomater. 2010:122-127.

11. Deog Gyu Seo Zirconia surface treatment for successful bonding. Restor Dent Endod. 2014 39(4): 333.

12. Ahn J, Yi Y, Lee Y, Seo D. Shear Bond Strength of MDPContaining Self-Adhesive Resin Cement and Y-TZP Ceramics: Effect of Phosphate Monomer-Containing Primers. Biomed Res Int. 2015;2015:9-11.
13. Yap AU, Wang X, Wu X, Chung SM. Comparative hardness and modulus of tooth-colored restoratives: A depth-sensing micro-indentation study. Biomaterials 2004, 25, 2179-2185.

14. Irving W, Road P. Bonding of Resin Materials to AllCeramics: A Review Liang Chen and Byoung In Suh Research and Development, Bisco Inc, 2012; 3(1):7-17.

15. Makramani BMA Al, Razak AAA, Hassan MIA, Sanabani FA Al, Albakri FM. Effect of Luting Cements On the Bond Strength to Turkom-Cera All-Ceramic Material. Open Access Maced J Med Sci. 2018;6(3):548-553.

16. Su N, Yue L, Liao Y, et al. The effect of various sandblasting conditions on surface changes of dental zirconia and shear bond strength between zirconia core and indirect composite resin. J Adv Prosthodont. 2015; 7(3):214-23.

17. Schelling PK, Phillpot SR, Wolf D. Mechanism of the Cubic to Tetragonal Phase Transition in Zirconia and YttriaStabilized Zirconia by Molecular-Dynamics Simulation. Journal of the American Society 2001; 84(7):1609-19.

18. Nagaoka N, Yoshihara K, Feitosa VP, et al. Chemical interaction mechanism of 10-MDP with zirconia. Nat Publ Gr. 2017:1-7. doi:10.1038/srep45563

19. Aboushelib, M. N. Evaluation of zirconia/resin bond strength and interface quality using a new technique. J. Adhes. Dent. 2011; 13: 255-60.

20. Wolfart M, Lehmann F, Wolfart S, Kern M. Durability of resin bond strength to zirconia ceramic after using different surface conditioning methods. Dent Mater 2007;23:45e50.

21. Hallmann L, Ulmer P, Reusser E, Ha“mmerle CH. Surface characterization of dental Y-TZP ceramic after air abrasion treatment. J Dent 2012;40:723-35.

22. Kulunk Ş, Kulunk T, Ural Ç, Kurt M, Baba S. Effect of air abrasion particles on the bond strength of adhesive resin cement to zirconia core. Acta Odontol Scand. 2011;69(2):88-94.

23. Cavalcanti AN, Foxton RM, Watson TF, Oliveira MT, Giannini M, Marchi GM. Bond Strength of Resin Cements to a Zirconia Ceramic with Different Surface Treatments. Oper Dent. 2009:280-287.

24. Yi YA, Ahn JS, Park YJ, Jun SH, Lee IB, Cho BH, Son HH, Seo DG The Effect of Sandblasting and Different Primers on Shear Bond Strength Between Yttria-tetragonal Zirconia Polycrystal Ceramic and a Self-adhesive Resin Cement. Oper Dent.2015:63-71. 